\title{
Apolipoprotein A5 regulates intracellular triglyceride metabolism in adipocytes
}

\author{
XIAO-YAN ZHENG ${ }^{*}$, BI-LIAN YU*, YU-FEI XIE, SHUI-PING ZHAO and CHEN-LU WU \\ Department of Cardiology, The Second Xiangya Hospital, Central South University, Changsha, Hunan 410011, P.R. China
}

Received July 27, 2016; Accepted June 22, 2017

DOI: $10.3892 / \mathrm{mmr} .2017 .7461$

\begin{abstract}
It has previously been demonstrated that apolipoprotein A5 (apoA5) can be internalized by human adipocytes and significantly decreases intracellular triglyceride content. In the present study, endocytosis of apoA5 by adipocytes under different conditions, and the underlying mechanism by which apoA5 regulates cellular triglyceride storage, was investigated. The results revealed that the apoA5 protein was detected in human subcutaneous abdominal adipose tissues. In addition, the uptake of apoA5 was attenuated in human obese adipose tissues and in cultured adipocytes with hypertrophy or insulin resistance. Low-density lipoprotein receptor protein 1 (LRP1) knockdown in adipocytes resulted in a decrease in internalized apoA5 content, suggesting that LRP1 serves a role in apoA5 uptake. Treatment of adipocytes with apoA5 decreased the expression of the lipid droplet-associated proteins such as cidec and perilipin. ApoA5-treated adipocytes demonstrated an increase in lipolysis activity and expression of uncoupling protein 1, which is the molecular effector of thermogenesis in brown adipocytes. These results suggested that decreased triglyceride accumulation in adipocytes induced by apoA5 may be associated with enhanced lipolysis and energy expenditure, which may result from reduced expression of cidec and perilipin. In conclusion, the present study demonstrated a novel role of apoA5 in regulating the intracellular triglyceride metabolism of adipocytes. The results of the present study suggested that apoA5 may serve as a potential therapeutic target for the treatment of obesity and its related disorders.
\end{abstract}

\section{Introduction}

Apolipoprotein A5 (apoA5), first described in 2001, has been demonstrated to be an important modulator of triglyceride (TG)

Correspondence to: Dr Shui-Ping Zhao, Department of Cardiology, The Second Xiangya Hospital, Central South University, 139 Renmin Road, Changsha, Hunan 410011, P.R. China

E-mail: zhaosp@medmail.com.cn

*Contributed equally

Key words: apolipoprotein A5, lipolysis, perilipin, cidec, uncoupling protein metabolism (1). ApoA5 is synthesized and secreted exclusively by the liver and is present in plasma associated with chylomicrons, very low density lipoprotein (LDL) and high density lipoprotein (1). Increasing evidence suggests that a number of variants of the gene encoding human apoA5 (APOA5) alter plasma TG levels and is associated with obesity (2-4). Lower plasma levels of apoA5 were observed in obese subjects and were inversely associated with body mass index (BMI) in humans, suggesting that decreased apoA5 levels may have importance in the pathophysiology of obesity in humans $(5,6)$. However, the underlying mechanisms of apoA5-associated obesity remain to be understood and cannot be fully explained by its impact on plasma TG levels $(7,8)$.

An intracellular role of apoA5 has been suggested, as apoA5 is associated with cytoplasmic lipid droplets $(9,10)$ and affects intrahepatic TG accumulation $(10,11)$. Given that adipocytes provide the largest storage depot for energy in the form of TG within lipid droplets, and serve an important role in the development of obesity, the present study hypothesized that apoA5 may additionally target adipocytes and regulate adipocyte lipid storage. Our previous data demonstrated that apoA5 may be internalized by human adipocytes, as treatment of adipocytes with receptor associated protein (RAP) or heparin, both of which interrupt binding of apoA5 to low-density lipoprotein receptor (LDLR) family members, resulted in a decrease in the quantity of apoA5 internalized by adipocytes (12). LDLR-related protein 1 (LRP1), which belongs to the LDLR family and is abundantly expressed in adipocytes, has been reported to interact with apoA5 with high affinity and mediates receptor-mediated endocytosis of apoA5 (13). This suggests that LRP1 may serve a role in uptake of apoA5 by adipocytes. In addition, it was revealed that internalized apoA5 surrounded lipid droplets, co-localized with the known lipid droplet protein perilipin and significantly decreased cellular TG content in adipocytes (12).

Lipid droplets are dynamic organelles that contain a neutral lipid core surrounded by a phospholipid monolayer, and is coated with specific proteins $(14,15)$. Numerous studies on the formation of lipid droplets and regulation of lipid accumulation have demonstrated the importance of lipid droplet-associated proteins in these processes. Based on shared sequence homology, one set of lipid droplet proteins, the perilipin-adipophilin-TIP47 (PAT) family of proteins, are well characterized (16). Within adipocytes, perilipin is the most abundant protein on adipocyte lipid droplets and 
is an important regulator of lipid storage and release. Under basal conditions, perilipin is a gatekeeper that prevents lipases from gaining access to neutral lipids in the droplet core, and therefore reduces TG hydrolysis (17). By contrast, once phosphorylated in response to catecholamine stimulation, perilipin actively facilitates lipase action, partially by recruiting hormone-sensitive lipase (HSL, officially known as LIPE) to the droplet surface. Ablation of perilipin from white adipose tissue (WAT) causes dysregulation of adipocyte lipid storage characterized by increased basal lipolysis and decreased stimulated lipolysis and results in a dramatic reduction in WAT mass $(18,19)$.

Cidec, a human homolog of rodent fat-specific protein 27 (Fsp27), has been identified as a novel lipid droplet-associated protein in adipocytes and serves an important role in controlling diverse metabolic processes (20-24). Cidec/Fsp27 belong to the cell death-inducing DNA fragmentation factor- $\alpha$-like effector (Cide) family that shares a common CIDE-N domain in the N-terminus and CIDE-C domain in the C-terminus (25). In humans, cidec is predominantly expressed in subcutaneous adipose tissue (26). Nishino et al (22) demonstrated that depletion of Fsp27 in murine white adipocytes resulted in the formation of numerous small lipid droplets, increased lipolysis and decreased TG storage, whereas expression of Fsp27 in COS monkey fibroblast cells promoted the formation of large lipid droplets. This suggested that Fsp27 contributes to efficient energy storage in WAT by promoting the formation of unilocular lipid droplets, thereby restricting lipolysis. In addition Fsp27-knockout mice have been described to exhibit a phenotype of obesity-resistance, elevated oxygen consumption, reduced WAT mass and smaller white adipocytes with multilocular lipid droplets $(22,23)$. In addition, expression of genes associated with fatty acid $\beta$-oxidation, mitochondrial biosynthesis and brown adipose tissue (BAT)-special genes were significantly increased in Fsp27 knockout mice. These alterations suggest that WAT tissue of Fsp27-knockout mice may have acquired certain BAT-like properties, and therefore was transformed from a typical energy storage tissue into an energy consuming tissue.

In the present study, expression of apoA5 by adipocytes under different conditions, the effect of apoA5 on the expression of the genes encoding perilipin and cidec and the mechanism responsible for apoA5 negative modulation in adipocyte TG accumulation was investigated.

\section{Materials and methods}

Materials. Dulbecco's modified Eagle's medium (DMEM)/F-12, DMEM, fetal bovine serum (FBS) and human insulin were all purchased from Invitrogen; Thermo Fisher Scientific, Inc. (Waltham, MA, USA). Dexamethasone (Dex), 3-isobutyl-1-methylxanthine (IBMX), rosiglitazone, isoproterenol and recombinant murine tumor necrosis factor- $\alpha$ (TNF- $\alpha$ ) were purchased from Sigma-Aldrich; Merck KGaA (Darmstadt, Germany). Mouse anti-human apoA5 antibody was obtained from Novus Biologicals, LLC (cat. no. NB400-139; Littleton, CO, USA) (6). Rabbit anti-mouse LRP1 antibody (cat. no. ab92544), mouse anti-human cidec antibody (cat. no. ab77115) and rabbit anti-human perilipin antibody (cat. no. ab50291) were all purchased from Abcam
(Cambridge, UK). Goat anti-rabbit IgG antibody (bs-0295G) and rabbit anti-mouse IgG antibody (bs-0296R) were purchased from Bioss (Beijing, China). Recombinant human apoA5 was produced in Escherichia coli and isolated, as previously described (27).

Samples of human tissues and cell lines. Subcutaneous adipose tissue samples were obtained from 19 patients (7 male and 12 female) undergoing elective open-abdominal surgical procedures in the Second Xiangya Hospital, Central South University, China between December 2014 and November 2015. None of the patients had known diabetes or severe systemic illness, and were not taking medications known to affect adipose tissue mass or metabolism. The mean BMI was $22.9 \mathrm{~kg} / \mathrm{m}^{2}$ (range, $16.3-31.1 \mathrm{~kg} / \mathrm{m}^{2}$ ), and the mean age was 40 years (range, 15-55). In particular, 3 obese patients (BMI $\geq 30$ ) were recruited together with 3 matched non-obese patients $(\mathrm{BMI}<25)$ to obtain the subcutaneous adipose tissues for comparing the endocytosis of apoA5 by adipocytes in vivo. Tissue samples were immediately either frozen in liquid nitrogen and stored at $-80^{\circ} \mathrm{C}$, or digested with collagenase (Gibco; Thermo Fisher Scientific, Inc.) to isolate preadipocytes, as previously described (28). The protocol was approved by the Ethics Committee of Central South University (Hunan, China) and all patients provided written informed consent. The 3T3-L1 preadipocyte murine cell line was obtained from the American Type Culture Collection (ATCC; Manassas, VA, USA).

Cell culture. Human preadipocytes were differentiated into adipocytes as previously described, with a few modifications (29). Briefly, human preadipocytes were cultured in DMEM/F-12 medium supplemented with 10\% FBS, $100 \mathrm{U} / \mathrm{ml}$ penicillin and $100 \mathrm{U} / \mathrm{ml}$ streptomycin (complete medium) and incubated at $37^{\circ} \mathrm{C}$ in a humidified $5 \% \mathrm{CO}_{2} / 95 \%$ air atmosphere. At $100 \%$ confluence, cells were treated with differentiation medium containing DMEM/F-12, $100 \mathrm{U} / \mathrm{ml}$ penicillin, $100 \mathrm{U} / \mathrm{ml}$ streptomycin, 5\% FBS, $1 \mu \mathrm{M}$ Dex, $500 \mu \mathrm{M}$ IBMX, $10 \mu \mathrm{g} / \mathrm{ml}$ insulin and $1 \mu \mathrm{M}$ rosiglitazone for 12 days. Cells continued to be cultured in differentiation medium, but without rosiglitazone for a further 9 days. Cells were then cultured in complete medium for 2 days. These cells were used as differentiated adipocytes in all experiments. Each medium was replaced with fresh medium every 3 days.

3T3-L1 fibroblasts were cultured and differentiated into adipocytes as previously described $(30,31)$. Cells at day 8 and day 21 post-induction of differentiation were used as non-hypertrophied and hypertrophied adipocytes, respectively (30). To render adipocytes insulin resistant, fully differentiated adipocytes were treated with $3 \mathrm{ng} / \mathrm{ml}$ recombinant murine TNF- $\alpha$ for 3 days, with daily replacement of medium.

Knockdown of LRP1 in adipocytes. Differentiated 3T3-L1 adipocytes were transfected with $5 \mathrm{nM}$ small interfering RNA (siRNA) targeting LRP1 (CGCTGACCCTATTTGAAGA) or non-silencing control siRNA (TTCTCCGAACGTGTC ACGT) using HiPerFect transfection reagent (Qiagen $\mathrm{GmbH}$, Hilden, Germany) for up to $48 \mathrm{~h}$ at $37^{\circ} \mathrm{C}$, according to the manufacturer's instructions. The silencing effect of LRP1 siRNA transfection was quantified by western blot analysis. 
Pulse-chase experiment. For expression studies, apoA5 was labeled with ${ }^{125} \mathrm{I}$ using Iodogen (Sigma-Aldrich; Merck KGaA) according to the manufacturer's instructions. Differentiated 3T3-L1 adipocytes were pulsed with ${ }^{125} \mathrm{I}$-labeled apoA5 $\left(\sim 2 \times 10^{6} \mathrm{cpm} /\right.$ well $)$ in serum-free medium for $2 \mathrm{~h}$ at $37^{\circ} \mathrm{C}$. At the end of the pulse, the medium was aspirated and cells were washed three times with ice-cold PBS, and incubated with heparin $(10 \mathrm{mg} / \mathrm{ml}$ in PBS; Sigma-Aldrich; Merck KGaA) for $3 \mathrm{~min}$ at room temperature, and then washed three times with PBS. Fresh medium was added, and cells were chased for various time periods $(0,1,2,4,6$ and $24 \mathrm{~h})$ at $37^{\circ} \mathrm{C}$. At the end of the chase period, the medium was collected and cells were treated with heparin as described above. This heparin wash was added to the collected medium which was then precipitated with $13 \%$ trichloroacetic acid to estimate degradation of apoA5. Following heparin treatment, cells were collected in $0.1 \mathrm{M} \mathrm{NaOH}$. Total cell protein was determined using the bicinchoninic acid assay (Novagen; Merck KGaA). Radioactivity (cpm) measured by gamma-ray counter was normalized to cell protein, and data are expressed as total $\mathrm{cpm} / \mathrm{mg}$ protein at each time-point.

Measurement of intracellular TG content and glycerol release. Differentiated human adipocytes were incubated in serum-free DMEM/F-12 in the presence of $200 \mathrm{ng} / \mathrm{ml}$ recombinant apoA5 for $48 \mathrm{~h}$. The TG content of adipocytes was determined using a triglyceride quantification kit (BioVision, Inc., Milpitas, CA, USA) according to the manufacturer's instructions. For lipolysis analysis, adipocytes were washed with PBS once to remove the phenol red. Then $200 \mathrm{ng} / \mathrm{ml}$ apoA5 was added in phenol red free DMEM/F-12 for $24 \mathrm{~h}$. In some experiments, after apoA5 treatment, adipocytes were subsequently incubated with $10 \mu \mathrm{M}$ isoproterenol for $3 \mathrm{~h}$. At the end of incubation, the medium was collected and glycerol content was measured using a colorimetric assay at $412 \mathrm{~nm}$ (BioVision, Inc.).

Oil Red $O$ staining for lipid droplets. Mature adipocytes grown on coverslips were washed three times with cold PBS, fixed in $4 \%$ paraformaldehyde for $20 \mathrm{~min}$ and permeabilized with $0.2 \%$ Triton X-100 in PBS for $10 \mathrm{~min}$ at room temperature. Oil Red O (0.5\% in isopropyl alcohol; Sigma-Aldrich; Merck $\mathrm{KGaA}$ ) was mixed with distilled water in the ratio of 60:40 and filtered through $0.45 \mu \mathrm{m}$ filters. The freshly prepared Oil Red $\mathrm{O}$ solution was added to permeabilized cells for $30 \mathrm{~min}$. These cells were then washed with PBS three times, incubated with $0.2 \mu \mathrm{g} / \mathrm{ml}$ of 4',6-diamidino-2-phenylindole for $10 \mathrm{~min}$ at room temperature and washed with PBS three times. Cells were mounted on slides with ProLong Gold antifade reagent (Invitrogen; Thermo Fisher Scientific, Inc.), and visualized under a confocal microscope (model TCS SP5; Leica Microsystems, Inc., Buffalo Grove, IL, USA). The size of Oil Red O-stained lipid droplets was calculated using ImageJ software version 1.48s (National Institutes of Health, Bethesda, MD, USA).

Western blot analysis. Total protein lysate from frozen tissues or cultured cells was prepared using lysis buffer containing $50 \mathrm{mM}$ Tris (pH 7.4), 150 mM NaCl, 1 mM EDTA, $1 \%$ Triton X-100, $0.5 \%$ sodium deoxycholate and protease inhibitor (Invitrogen;
Thermo Fisher Scientific, Inc.) on ice for $0.5 \mathrm{~h}$. The cell lysates were centrifuged at $13,000 \mathrm{x}$ g for $10 \mathrm{~min}$ at $4^{\circ} \mathrm{C}$. The supernatants were separated by $12 \%$ SDS-PAGE and transferred onto polyvinylidene difluoride membranes. The membranes were blocked for $2 \mathrm{~h}$ with $5 \%$ skim milk in TBS containing $0.1 \%$ Tween-20 at room temperature and incubated overnight at $4^{\circ} \mathrm{C}$ with mouse anti-human apoA5 $(1: 1,000)$, mouse anti-LRP1 $(1: 1,000)$, human anti-Cidec $(1: 1,000)$ or human anti-perilipin $(1: 1,000)$. The blots were then incubated with a corresponding horseradish peroxidase-conjugated secondary antibody (goat anti-rabbit IgG, 1:5,000 or rabbit anti-mouse $\mathrm{IgG}, 1: 5,000)$ for $1 \mathrm{~h}$ at room temperature. The immunoblot signals were visualized using an enhanced chemiluminescence substrate (Invitrogen; Thermo Fisher Scientific, Inc.) and quantified by Image J software version 1.48s (National Institutes of Health).

Reverse transcription-quantitative polymerase chain reaction $(R T-q P C R)$. Total RNA from human differentiated adipocytes was isolated using TRIzol reagent (Invitrogen; Thermo Fisher Scientific, Inc.) according to the manufacturer's instructions. First strand cDNA was synthesized using a cDNA synthesis kit (Fermentas; Thermo Fisher Scientific, Inc.) according to the manufacturer's instructions. qPCR was performed on an ABI 7300 Real-Time PCR instrument (Applied Biosystems; Thermo Fisher Scientific, Inc.) with SYBR-Green mix (Takara Bio Inc., Otsu, Japan) and specific primers to amplify the genes. An initial denaturation step at $95^{\circ} \mathrm{C}$ for $30 \mathrm{~min}$; 40 cycles at $95^{\circ} \mathrm{C}$ for $5 \mathrm{sec}$ and $60^{\circ} \mathrm{C}$ for $31 \mathrm{sec}$. Gene expression levels of the genes encoding cidec (CIDEC), perilipin (PLIN1), diacylglycerol acyltransferase (DGAT) 1, DGAT2, stearoyl-CoA desaturase 1 (SCD1), HSL, adipose triglyceride lipase (ATGL), nuclear respiratory factor $1(N R F 1)$, subunit IV of cytochrome $c$ oxidase (COXIV, officially known as COX4II), PPAR $\gamma$ coactivator $1 \alpha(P G C 1 \alpha)$, uncoupling protein $1(U C P 1)$ and forkhead box $\mathrm{C} 2$ (FOXC2), were normalized to $18 \mathrm{~S}$ rRNA, and presented as the fold change relative to the control. The following primer sequences were used: $C I D E C$ sense, 5'-AAGTCCCTTAGC CTTCTCTACC-3' and antisense, 5'-CCTTCCTCACGCTTC GATCC-3'; PLIN1 sense, 5'-GACCTCCCTGAGCAGGAG AAT-3' and antisense, 5'-GTGGGCTTCCTTAGTGCTGG-3'; DGAT1 sense, 5'-AACCTCATCAAGTATGGCATCCT-3' and antisense, 5'-CATTGGCCGCAATAACCAGG-3'; DGAT2 sense, 5'-AAGACCCTCATAGCCGCCTA-3' and antisense, 5'-TTGGACCTATTGAGCCAGGTG-3'; SCD1 sense, 5'-TCT AGCTCCTATACCACCACCA-3' and antisense, 5'-TGTCGT CTTCCAAGTAGAGGG-3'; HSL sense, 5'-TCAGTGTCTAG GTCAGACTGG-3' and antisense, 5'-AGGCTTCTGTTGGGT ATTGGA-3'; ATGL sense, 5'-ATGGTGGCATTTCAGACA ACC-3' and antisense, 5'-CGGACAGATGTCACTCTCGC-3'; NRF1 sense, 5'-AACAAAATTGGGCCACGTTACA-3' and antisense, 5'-TCTGGACCAGGCCATTAGCA-3'; COXIV sense, 5'-CAGGGTATTTAGCCTAGTTGGC-3' and antisense, 5'-GCCGATCCATATAAGCTGGGA-3'; PGC1 $\alpha$ sense, 5'-TCTGAGTCTGTATGGAGTGACAT-3' and antisense, 5'-CCAAGTCGTTCACATCTAGTTCA-3'; UCP1 sense, 5'-AGGTCCAAGGTGAATGCCC-3' and antisense, 5'-GCG GTGATTGTTCCCAGGA-3'; FOXC2 sense, 5'-CCTCCTGG TATCTCAACCACA-3' and antisense, 5'-GAGGGTCGAGTT CTCAATCCC-3'; 18S rRNA sense, 5'-GCTTAATTTGAC TCAACACGGGA-3' and antisense, 5'-AGCTATCAATCT 


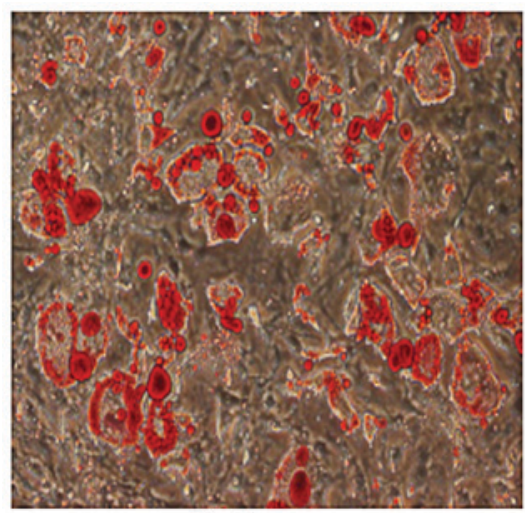

Day 8

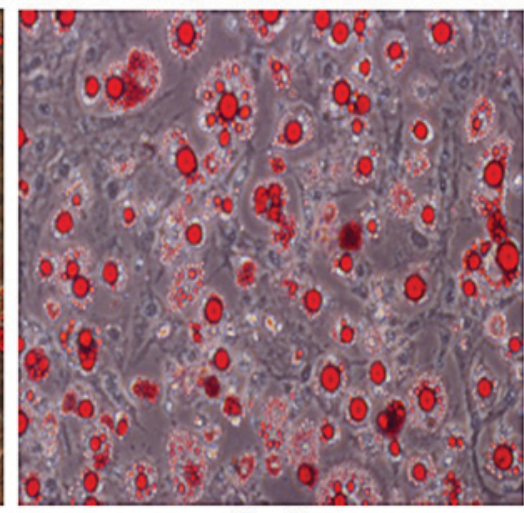

Day 21

Figure 1. Morphological alterations in 3T3-L1 adipocytes during adipocyte hypertrophy. Oil red O staining was performed at day 8 (non-hypertrophied cells) and day 21 (hypertrophied cells). Original magnification, x200.

GTCAATCCTGTC-3'. Gene expression levels were quantified using the $2^{-\triangle \triangle C q}$ method and normalized to the internal reference gene $18 \mathrm{~S}$ rRNA (32).

Statistical analysis. Statistical comparisons between 2 groups were assessed by a two-tailed, unpaired Student's t-test. Comparisons between $\geq 3$ groups were analyzed by one-way analysis of variance followed by a Newman-Keuls post hoc test. $\mathrm{P}<0.05$ was considered to indicate a statistically significant difference. Data are expressed as the mean \pm standard error of $\geq 3$ independent experiments.

\section{Results}

Decreased endocytosis of apoA5 by adipocytes in obese and insulin resistant states. 3T3-L1 fibroblasts were completely differentiated into adipocytes after 8 days incubation with induction media. For generating hypertrophied adipocytes, 3T3-L1 adipocytes were cultured up to 21 days after the induction of differentiation. Oil red $\mathrm{O}$ staining exhibited a gradual increase in lipid accumulation from day 8 to day 21 (Fig. 1). At $4 \mathrm{~h}$ after the addition of apoA5, internalized apoA5 was quantified by immunoblotting. During the course of adipocyte hypertrophy, apoA5 internalization was significantly lower on day $21(15 \%)$ in hypertrophied cells than that on day 8 in non-hypertrophied control cells (Fig. 2A). Using a pulse-chase protocol in which cells were incubated with $\left[{ }^{125} \mathrm{I}\right]$-labeled apoA5 and chased for various time-points up to $24 \mathrm{~h}$ after removal of the radiolabeled sample, internalized, labeled apoA5 in hypertrophied adipocytes was significantly decreased by $66 \%$ compared with control cells (Fig. 2B). The quantity of degraded apoA5 in the medium was increased in a time-dependent manner during the chase period, reaching $63 \%$ of total apoA5 at the $24 \mathrm{~h}$ time-point both in hypertrophied and non-hypertrophied adipocytes (data not shown).

Similar results were observed in the case of the insulin-resistant adipocytes. An in vitro model of metabolic insulin resistance was established using TNF- $\alpha$-treated 3T3-L1 adipocytes, as described previously (33). Western blot analysis revealed that TNF- $\alpha$ treatment decreased apoA5 internalization by $52 \%(\mathrm{P}<0.05$; Fig. $3 \mathrm{~A})$. Similar data was also confirmed by a pulse-chase experiment (Fig. 3B).
A
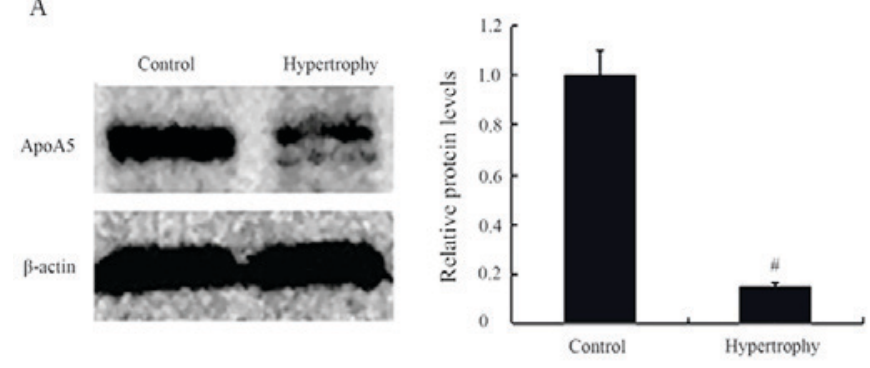

B

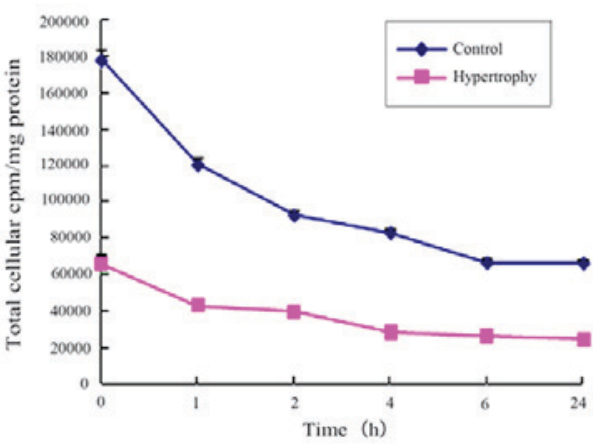

Figure 2. Endocytosis of apoA5 in hypertrophied adipocytes (21 days of differentiation) is reduced compared with the non-hypertrophied control (8 days of differentiation). (A) Western blot analysis of apoA5 expression in hypertrophied adipocytes. Differentiated 3T3-L1 adipocytes at day 8 and day 21 were starved in serum-free medium for $12 \mathrm{~h}$. Cells were then incubated in serum-free medium in the presence of $600 \mathrm{ng} / \mathrm{ml}$ recombinant human apoA5 for $4 \mathrm{~h}$. $\beta$-actin served as a loading control. ${ }^{"} \mathrm{P}<0.05$ vs. control. (B) Differentiated 3T3-L1 adipocytes at day 8 and 21 were pulsed with ${ }^{125} \mathrm{I}$-apoA5 for $2 \mathrm{~h}$ in serum-free medium and chased for various time-points up to $24 \mathrm{~h}$. Radioactivity (cpm) was normalized to cell protein, and data are expressed as total $\mathrm{cpm} / \mathrm{mg}$ protein at each time-point. Data are expressed as the mean \pm standard error of 3 independent experiments. ApoA5, apolipoprotein A5.

To determine the impact of obesity on apoA5 expression in human adipose tissue, we obtained subcutaneous abdominal adipose tissue biopsies from 6 patients with a wide range of BMI. The apoA5 protein was detected in these human adipose tissue samples. The amount of apoA 5 was significantly reduced by $69 \%$ in the obese group as compared with the non-obese group (Fig.4).

Silencing of LRP1 expression inhibits apoA5 expression by adipocytes. It was investigated whether knockdown of LRP1 
A
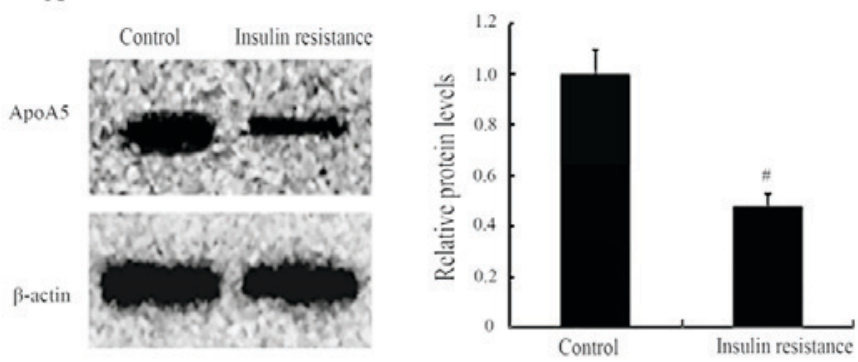

B

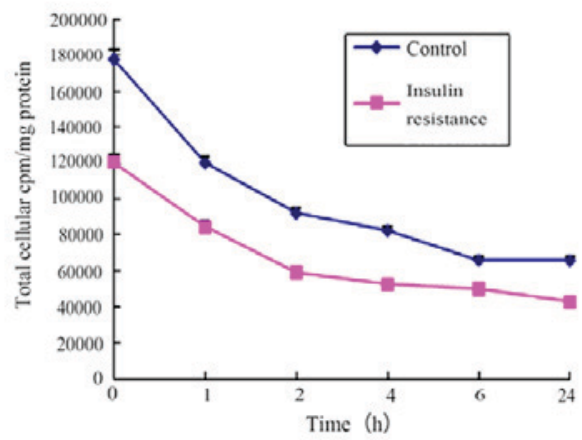

Figure 3. Expression of apoA5 by adipocytes in an insulin-resistant state. Fully differentiated 3T3-L1 adipocytes for 8 days were treated for 3 days with PBS (control adipocytes) or with $3 \mathrm{ng} / \mathrm{ml}$ recombinant murine TNF- $\alpha$ to render them insulin resistant. (A) Western blot analysis of the uptake of apoA5 in control and insulin-resistant adipocytes following incubation with $600 \mathrm{ng} / \mathrm{ml}$ recombinant human apoA5 for 4 hrs. $\beta$-actin served as a loading control. ${ }^{\text {P }} \mathrm{P}<0.05$ vs. control. (B) Normal and insulin-resistant adipocytes were pulsed with ${ }^{125} \mathrm{I}$-apoA 5 for $2 \mathrm{~h}$ in serum-free medium and chased for various times after various time-points up to $24 \mathrm{~h}$. Radioactivity (cpm) was normalized to cell protein, and data are expressed as total $\mathrm{cpm} / \mathrm{mg}$ protein at each time-point. Data are expressed as the mean \pm standard error of 3 independent experiments. ApoA5, apolipoprotein A5.
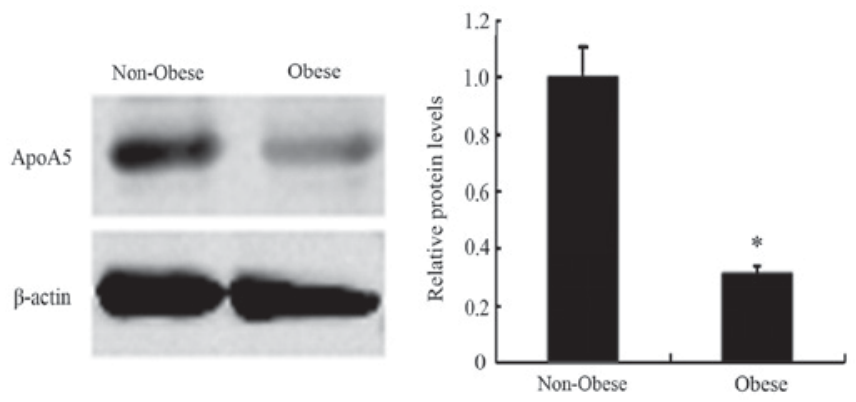

Figure 4. ApoA5 protein levels in obese and non-obese human adipose tissue. Western blot analysis of apoA5 in subcutaneous abdominal adipose tissue from obese $(B M I \geq 30)(n=3)$ and non-obese (BMI <25) humans $(n=3)$. The experiment was performed three times. ${ }^{*} \mathrm{P}<0.05$ vs. control. ApoA5, apolipoprotein A5.

expression in adipocytes prevents apoA5 internalization. After $48 \mathrm{~h}$ transfection of differentiated 3T3-L1 adipocytes with LRP1 siRNA, knockdown efficiency was $87 \%(\mathrm{P}<0.05)$ as determined by western blot analysis (Fig. 5A). The reduction of LRP1 expression resulted in decreased apoA5 uptake by adipocytes $(\sim 70 \%)$, as determined by western blot analysis and the pulse-chase experiment (Fig. 5B and C).

ApoA5 significantly decreases TG accumulation in adipocytes but does not affect lipid droplet formation. Our previous
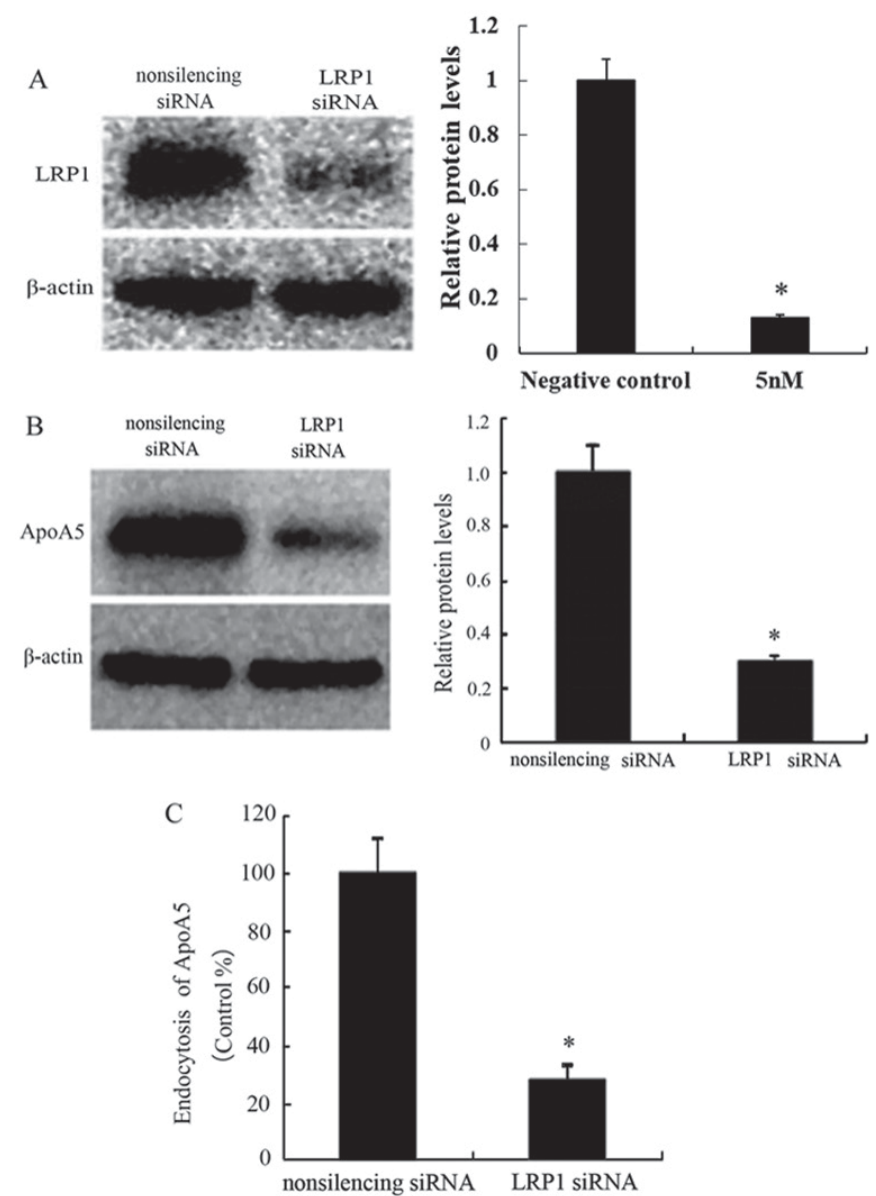

Figure 5. Expression of apoA5 by adipocytes following LRP1 siRNA transfection. (A) Efficiency of LRP1 siRNA knockdown as determined by western blot analysis. ${ }^{*} \mathrm{P}<0.05$ vs. control. (B) Western blot analysis of apoA5 protein expression in differentiated 3T3-L1 adipocytes transfected with LRP1 siRNA or nonsilencing siRNA following incubation with $600 \mathrm{ng} / \mathrm{ml}$ recombinant human apoA5 for $4 \mathrm{~h}$. "P<0.05 vs. control. (C) Transfected cells were pulsed with ${ }^{125} \mathrm{I}$-apoA5 for $2 \mathrm{~h}$ in serum-free medium and collected at the end of pulse period. ${ }^{*} \mathrm{P}<0.05$ vs. control. Data are expressed as the mean \pm standard error of 3 independent experiments. ApoA5, apolipoprotein A5; LRP1, low-density lipoprotein receptor protein 1; siRNA, small interfering RNA.

study revealed that treatment with 200 and $600 \mathrm{ng} / \mathrm{ml}$ apoA5 for $48 \mathrm{~h}$ significantly decreased intracellular TG levels in human adipocytes (12). As $200 \mathrm{ng} / \mathrm{ml}$ is within the normal range of human plasma levels of apoA5 $(5.4-455.6 \mathrm{ng} / \mathrm{ml})(6)$, this concentration was used in the present study. ApoA5 treatment for $48 \mathrm{~h}$ led to a significant decrease in TG content in adipocytes, which was consistent with our previous findings (6) $(\mathrm{P}<0.001$; Fig. 6A). Next, the effect of apoA5 on the morphological changes of lipid droplets was investigated. Oil Red $\mathrm{O}$ staining and confocal microscopy revealed that apoA5 treatment did not affect lipid droplet formation in adipocytes; however, the lipid droplets in apoA5-treated cells were generally smaller than those in control cells (Fig. 6B and C).

ApoA5 markedly decreases the expression of the genes encoding cidec and perilipin in adipocytes. RT-qPCR analysis revealed that apoA5 decreased CIDEC mRNA expression levels in a time-dependent manner with maximal effect at $48 \mathrm{~h}$ (Fig. 7A). Treatment with apoA5 for $48 \mathrm{~h}$ decreased CIDEC mRNA expression levels by $71 \%$. Similarly, apoA 5 significantly 

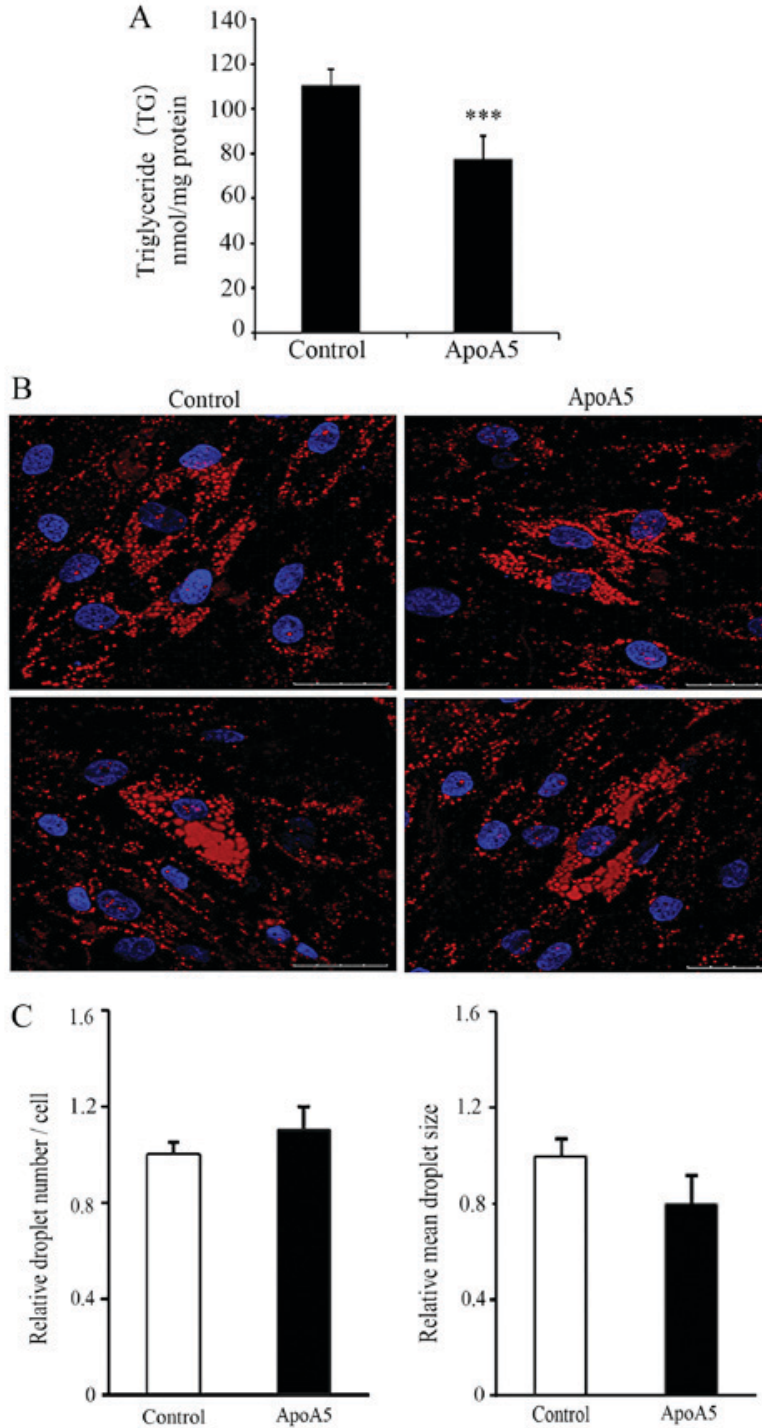

Figure 6. ApoA5 treatment reduces the TG concentration but did not affect lipid droplet formation in human adipocytes. Differentiated adipocytes were starved in serum-free medium for $12 \mathrm{~h}$. Cells were then incubated in serum-free medium in the presence or absence of $200 \mathrm{ng} / \mathrm{ml}$ apoA5 for $48 \mathrm{~h}$. (A) TG concentration in adipocytes was determined. ${ }^{* * *} \mathrm{P}<0.001$ vs. control. (B) Cells were stained with Oil Red O and 4',6-diamidino-2-phenylindole. Representative images from confocal microscopy analysis are presented. Scale bar, $30 \mu \mathrm{m}$. (C) Quantification of droplet number per cell (left graph) and mean droplet size (right graph) after treatment with or without apoA5. Data are expressed as the mean \pm standard error of at 5 independent experiments. ApoA5, apolipoprotein A5; TG, triglyceride.

decreased PLIN1 mRNA expression levels by $45 \%$, with maximal effect at $24 \mathrm{~h}$ (Fig. 7B). Western blot analysis further confirmed that apoA5 reduced the protein expression levels of cidec and perilipin in human adipocytes (Fig. 7C).

\section{ApoA5 treatment results in enhanced lipolysis and upregulated} expression of the brown fat-specific gene UCP1. To further address the potential regulation of TG-associated metabolic processes by apoA5, lipolysis in adipocytes was measured following apoA5 treatment. Measurement of glycerol released into the medium during culture of cells under basal conditions for $24 \mathrm{~h}$ revealed that lipolysis was significantly increased in apoA5-treated adipocytes. Examination of lipolysis in
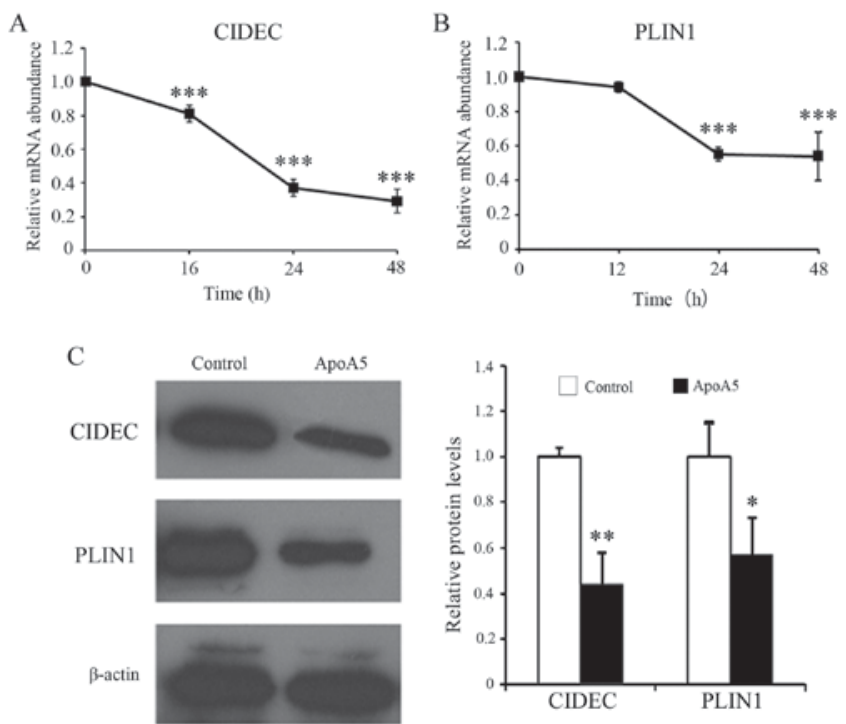

Figure 7. ApoA5 reduces the expression levels of the genes encoding cidec and perilipin in human adipocytes. Differentiated adipocytes were starved in serum-free medium for $12 \mathrm{~h}$. Cells were then incubated in serum-free medium in the presence or absence of $200 \mathrm{ng} / \mathrm{ml}$ apoA5 for various time-points up to 48 h. mRNA expression levels of (A) CIDEC and (B) PLIN1. The mRNA expression levels of each gene normalized relative to 18S rRNA expression, and presented as mRNA levels relative to the $0 \mathrm{~h}$ control (without apoA5). ${ }^{* * * *} \mathrm{P}<0.001$ vs. control. (C) Western blot and densitometric analysis of cidec, perilipin and $\beta$-actin (control) expression levels in adipocytes $24 \mathrm{~h}$ after $200 \mathrm{ng} / \mathrm{ml}$ apoA5 treatment. Data are expressed as the mean \pm standard error of 3 independent experiments. ${ }^{*} \mathrm{P}<0.05$ and ${ }^{* *} \mathrm{P}<0.01$ vs. control. ApoA5, apolipoprotein A5; CIDEC, cidec gene; PLIN1, perilipin gene.

response to $\beta$-adrenergic stimulation revealed that treatment with apoA5 also enhanced isoproterenol-induced lipolysis in human adipocytes (Fig. 8).

Next, the effects of apoA5 on expression of genes associated with lipid synthesis and hydrolysis in adipocytes was investigated. RT-qPCR analysis was performed $48 \mathrm{~h}$ after intervention of apoA5 and revealed that the mRNA expression levels of DGAT1, DGAT2 and SCD1, all of which participate in lipid synthesis, did not significantly differ between apoA5-treated and control adipocytes (Fig. 9A). In addition, mRNA expression levels of $H S L$ and $A T G L$, both of which contribute to TG hydrolysis in adipocytes, were not significantly different between the apoA5-treated and control cells (Fig. 9B). In addition, the expression of genes associated with mitochondrial biogenesis or oxidative phosphorylation were measured. Treatment of apoA5 for $48 \mathrm{~h}$ did not affect the mRNA expression levels of NRF1, COXIV and PGCl $\alpha$ in adipocytes (Fig. 9C). Finally, the mRNA expression levels of brown fat-specific gene UCP1 were measured, which serves a role in uncoupling oxidative phosphorylation and converts this proton gradient energy into heat to maintain normal body temperature (34). UCPl mRNA expression levels were significantly increased $(\sim 1.87$-fold; $\mathrm{P}<0.05)$ by apoA5 treatment for $48 \mathrm{~h}$ (Fig. 9D). The mRNA expression levels of FOXC2, which is an important regulator of UCPl (35), were also significantly elevated $(\mathrm{P}<0.05$; Fig. $\mathrm{D})$. These results suggested that the decrease in TG accumulation in human adipocytes after apoA5 treatment may be accompanied by increased lipolysis, uncoupling or thermogenesis, but not by increased mitochondrial biogenesis or oxidative phosphorylation. 


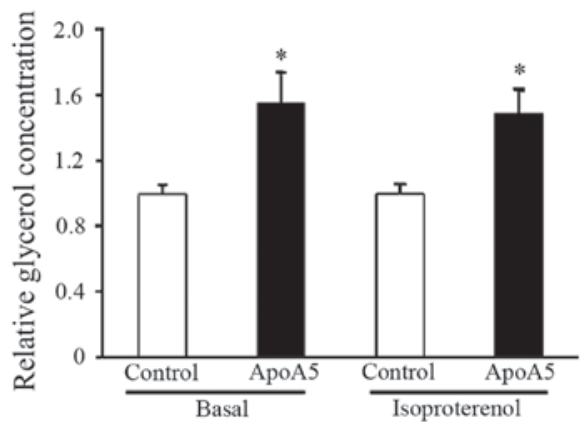

Figure 8. Effects of apoA5 treatment on glycerol concentration in human adipocytes. Differentiated adipocytes were incubated in serum-free medium in the presence or absence of $200 \mathrm{ng} / \mathrm{ml}$ apoA 5 for $24 \mathrm{~h}$. Cells were then incubated for $3 \mathrm{~h}$ in the absence (basal) or presence of $10 \mu \mathrm{M}$ isoproterenol, after which concentrations of glycerol in the culture medium were measured. Data are expressed relative to the value for control cells, and are expressed as the mean \pm standard error of 3 independent experiments. ${ }^{*} \mathrm{P}<0.05$ vs. control. ApoA5, apolipoprotein A5.

\section{Discussion}

The present study revealed that apoA5 protein was detected in human subcutaneous abdominal adipose tissues ex vivo, indicating that circulating apoA5 may be internalized by adipocytes in vivo. This finding concurs with our previous results in vitro (12). It was demonstrated that apoA5 expression was significantly decreased in both adipose tissue from obese patients and hypertrophied or insulin resistant 3T3-L1 adipocytes. The underlying mechanism for this phenomenon is unknown but it is possibly due to reduced uptake of apoA5, as the degraded rates of the apoA5 protein was similar in adipocytes under different conditions, as revealed by the pulse-chase experiment. The present study demonstrated that knockdown of LRP1 by siRNA transfection in adipocytes resulted in decreased apoA5 expression, suggesting that LRP1 serves a role in apoA5 uptake by adipocytes. As LRP1 has been demonstrated to be upregulated in obese mouse adipocytes and obese human adipose tissues (36), the decreased uptake of apoA5 is possibly due to reduced endocytic activity of LRP1. Hypertrophied adipocytes usually exhibit modified metabolic properties and develop insulin resistance (37). Under physiological conditions, insulin mediates recycling of LRP1 from an endosomal pool to the plasma membrane in differentiated adipocytes, resulting in an increase in the cell surface presentation of LRP1 and a concomitant increased endocytic activity $(38,39)$. Taken together, it may be hypothesized that, during insulin resistance, the movement of LRP1 from intracellular structures to the cell surface is attenuated, leading to decreased apoA5 endocytosis by adipocytes.

It was previously demonstrated that internalized apoA5 by human adipocytes was associated with lipid droplets and decreased cellular TG content (12). In the present study, the underlying mechanism of adipocytes regulation and TG storage was investigated. The results revealed that treatment with recombinant apoA5 significantly decreased the expression of the genes encoding cidec and perilipin, both of which localize to the surface of lipid droplets and serve roles in the negative regulation of lipolysis and promotion of TG accumulation in adipocytes. ApoA5-treated adipocytes exhibited
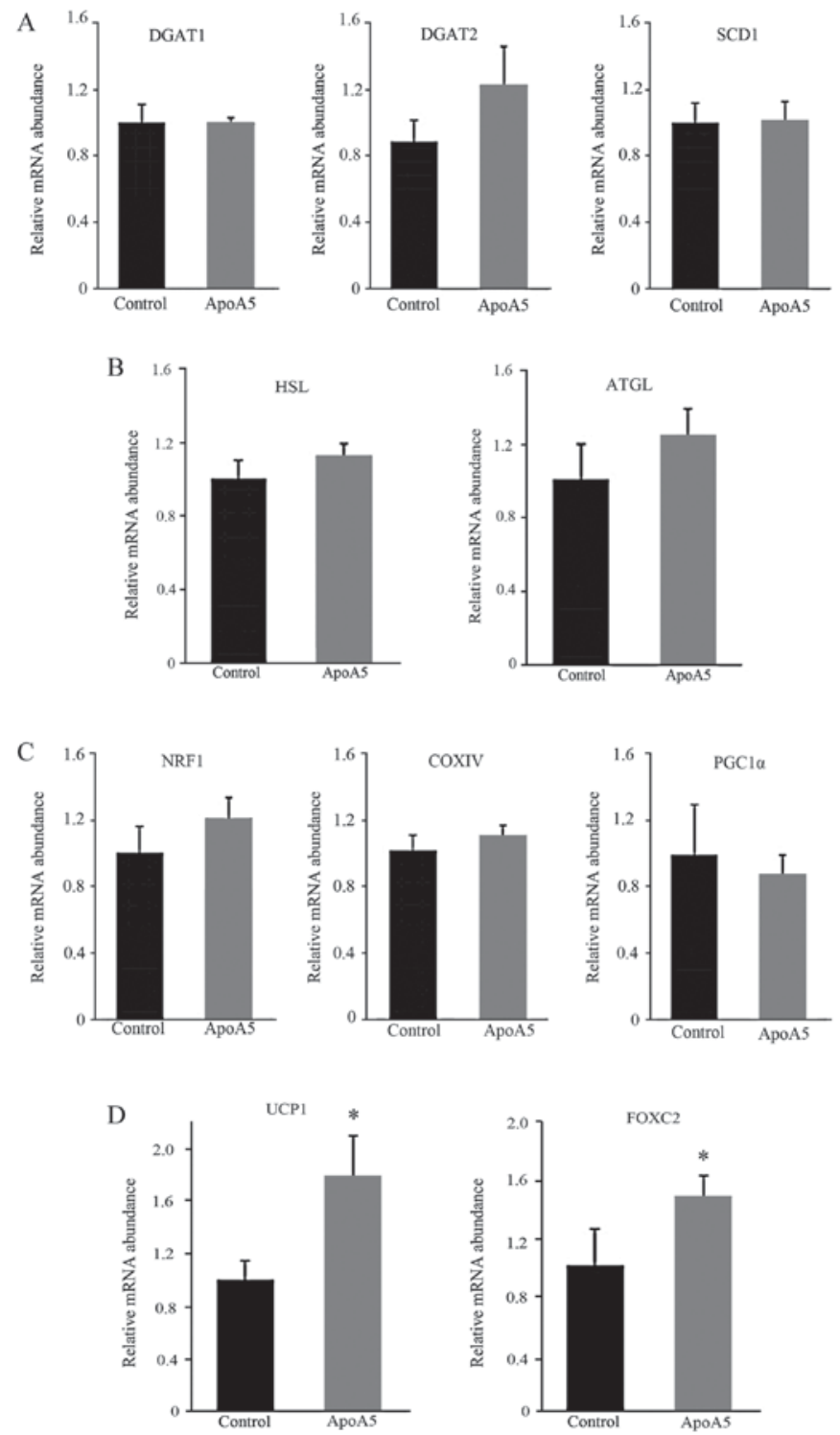

Figure 9. Effects of apoA5 treatment on the expression of lipid synthesis-associated genes in human adipocytes. mRNA expression levels of (A) lipolysis-associated genes, (B) TG hydrolysis-associated genes, (C) mitochondrial biogenesis or oxidative phosphorylation-associated genes and (D) BAT-specific genes in human adipocytes, $48 \mathrm{~h}$ after $200 \mathrm{ng} / \mathrm{m}$ apoA5 treatment. Data were normalized to $18 \mathrm{~S}$ rRNA mRNA expression levels, and are expressed as the mean \pm standard error of 5 independent experiments. ${ }^{*} \mathrm{P}<0.05$ vs. control. ApoA5, apolipoprotein A5; DGAT, diacylglycerol acyltransferase; SCD1, stearoyl-CoA desaturase 1; TG, triglyceride HSL, hormone-sensitive lipase; ATGL, adipose triglyceride lipase; NRF1, nuclear respiratory factor 1; COXIV, subunit IV of cytochrome $c$ oxidase; PGC1a, PPAR $\gamma$ coactivator $1 \alpha$; UCP1, uncoupling protein 1 ; FOXC2, forkhead box C2; BAT, brown adipose tissue.

increased lipolysis activity. This data concurred with observations of Nishino et al (22), who revealed that depletion of cidec or perilipin by siRNA transfection in cultured adipocytes enhanced basal and isoproterenol-induced lipolysis, whereas depletion of cidec and perilipin increased basal and stimulated lipolysis in an approximately additive manner. These results suggested that the reduced expression of cidec and perilipin induced by apoA5 may contribute individually to enhanced lipolysis activity. However, isolated adipocytes of perilipin null mice have been demonstrated to exhibit attenuated stimulated lipolytic activity and elevated basal lipolysis $(18,19)$. This 
discrepancy may be due to incomplete depletion of perilipin in the present study and the study by Nishino et al (22), as the remaining perilipin may still facilitate lipase action in response to catecholamine stimulation.

In addition to alterations in lipid accumulation and enhanced lipolysis, cidec/Fsp27 deficient adipocytes acquire a BAT-like property, including the formation of multiple small lipid droplets, an elevated rate of lipid oxidation, upregulated mitochondrial activity and expression of BAT-specific gene UCP1 $(22,23)$, which is not observed in perilipin depleted adipocytes. FOXC2, a crucial regulator of the cyclin adenosine monophosphate pathway that promotes BAT differentiation, has also been demonstrated to increase in the WAT of Fsp27-knockout mice (23). Additionally, Sawada et al (40) revealed that perilipin overexpression causes a significant reduction in Fsp27 (82\% reduction) which is associated with decreased adipocyte size, upregulation of UCP1 and genes involved in fatty acid oxidation and a decrease in lipogenic genes, suggesting that attenuation of Fsp27 is crucial in mediating these metabolic processes. Consistent with these previous observations, the present study revealed that the downregulation of cidec by apoA5 treatment was accompanied by increased gene expression levels of $U C P 1$ and $F O X C 2$ in human adipocytes, suggesting that apoA5 may upregulate these genes by decreasing cidec expression. Enhanced $F O X C 2$ expression may contribute to the increased expression of $U C P 1$. However, alterations in expression of genes associated with fatty acid oxidation, mitochondrial biosynthesis and lipid droplet formation, including DGAT1, DGAT2, SCD1, HSL, ATGL, NRF1, COXIV and PGCl $\alpha$, was not observed. As the present study only observed a $71 \%$ reduction of cidec induced by apoA5, it is possible that this may be due to incomplete depletion of cidec, which requires clarification with future research.

UCP1 is an inner mitochondrial membrane protein that may dissipate mitochondrial membrane potential, partially uncouple substrate oxidation and oxidative phosphorylation and promote the dissipation of cellular biochemical energy as heat to maintain body temperature (41). In particular, UCP1 is enriched in BAT and is minimally present in WAT (42). Strategies aimed at increasing UCP1 expression in adipose cells are of great interest in view of evidence that ectopic expression of UCP1 is significantly associated with a reduction in TG content in adipocytes or adipose tissue (43-45), and that a reduced brown adipose-like phenotype in WAT depots may contribute to obesity and type 2 diabetes in humans $(46,47)$. Therefore, alteration of energy balance via an increased utilization of fat in WAT may be a promising target for the treatment of obesity and metabolic syndrome. In the present study, in the presence of apoA5, the mRNA expression levels of UCPI were significantly increased in differentiated human adipocytes. Although 2 -fold increase in UCP1 induced by apoA5 is relatively mild, this small alteration in UCP1 expression may contribute to efficient energy dissipation and decreased TG accumulation. Toh et al (23) demonstrated that depletion of Fsp27 in differentiated mouse embryonic fibroblasts causes increased expression levels of UCP1 by $\sim 2$-fold, and is accompanied by reduced intracellular TG content. Consistent with this in vitro data, a clinical study by Oberkofler et al (46) also revealed that lean subjects have $\sim 2$-fold increased UCP1
mRNA expression levels in the intraperitoneal adipose tissue compared with that of obese groups. The present study revealed that apoA5 may behave as a regulator to promote $U C P 1$ gene expression in human adipocytes, suggesting that apoA5 may exert its protection against obesity by inducing white adipocytes to acquire certain characteristics of brown adipocytes and increasing energy consumption.

In conclusion, the present study suggested that apoA5 may be internalized by adipocytes both in vivo and in vitro, primarily via binding to LRP1. The uptake of apoA5 was attenuated in human obese adipose tissues and in cultured adipocytes with hypertrophy or insulin resistance. In addition, decreased TG accumulation in human adipocytes induced by apoA5 intervention may be associated with enhanced lipolysis and energy expenditure, which may result from reduced expression of cidec and perilipin. These findings may provide a greater understanding of the roles of apoA5 in regulating the intracellular TG metabolism of adipocytes. Under hypertrophied and insulin resistant conditions, attenuated endocytosis of apoA 5 by adipocytes may lead to excessive augmentation of TG storage and abnormal metabolism of adipocytes, which promotes the development of obesity. As a novel regulator of lipid storage in adipocytes, apoA5 may serve an important role in whole body energy homeostasis and may be a potential therapeutic target for the treatment of obesity and obesity-associated disorders.

\section{Acknowledgements}

The present study was supported by the National Natural Science Foundation of China (grant no. 81170262 and 81300205) and Specialized Research Fund for the Doctoral Program of Higher Education (grant no. 20130162120057).

\section{References}

1. Wong K and Ryan RO: Characterization of apolipoprotein A-V structure and mode of plasma triacylglycerol regulation. Curr Opin Lipidol 18: 319-324, 2007.

2. Horvatovich K, Bokor S, Baráth A, Maász A, Kisfali P, Járomi L, Polgár N, Tóth D, Répásy J, Endreffy E, et al: Haplotype analysis of the apolipoprotein A5 gene in obese pediatric patients. Int $\mathrm{J}$ Pediatr Obes 6: e318-e325, 2011.

3. Niculescu LS, Fruchart-Najib J, Fruchart JC and Sima A: Apolipoprotein A-V gene polymorphisms in subjects with metabolic syndrome. Clin Chem Lab Med 45: 1133-1139, 2007.

4. Zheng XY, Zhao SP and Yan H: The role of apolipoprotein A5 in obesity and the metabolic syndrome. Biol Rev Camb Philos Soc 88: 490-498, 2013.

5. Huang XS,Zhao SP,Hu M,Bai L, Zhang Q and Zhao W: Decreased apolipoprotein A5 is implicated in insulin resistance-related hypertriglyceridemia in obesity. Atherosclerosis 210: 563-568, 2010.

6. Zhao SP, Hu S, Li J, Hu M, Liu Q, Wu LJ and Zhang T: Association of human serum apolipoprotein A5 with lipid profiles affected by gender. Clin Chim Acta 376: 68-71, 2007.

7. Corella D, Lai CQ, Demissie S, Cupples LA, Manning AK, Tucker KL and Ordovas JM: APOA5 gene variation modulates the effects of dietary fat intake on body mass index and obesity risk in the Framingham Heart Study. J Mol Med (Berl) 85: $119-128,2007$.

8. Martin S, Nicaud V, Humphries SE and Talmud PJ; EARS group: Contribution of APOA5 gene variants to plasma triglyceride determination and to the response to both fat and glucose tolerance challenges. Biochim Biophys Acta 1637: 217-225, 2003.

9. Shu X, Chan J, Ryan RO and Forte TM: Apolipoprotein A-V association with intracellular lipid droplets. J Lipid Res 48: 1445-1450, 2007. 
10. Shu X, Nelbach L, Ryan RO and Forte TM: Apolipoprotein A-Y associates with intrahepatic lipid droplets and influences triglyceride accumulation. Biochim Biophys Acta 1801: 605-608, 2010.

11. Ress C, Moschen AR, Sausgruber N, Tschoner A, Graziadei I, Weiss H, Schgoer W, Ebenbichler CF, Konrad RJ, Patsch JR, et al: The role of apolipoprotein A5 in non-alcoholic fatty liver disease. Gut 60: 985-991, 2011.

12. Zheng XY,Zhao SP, Yu BL, Wu CL and Liu L: Apolipoprotein A5 internalized by human adipocytes modulates cellular triglyceride content. Biol Chem 393: 161-167, 2012.

13. Nilsson SK, Christensen S, Raarup MK, Ryan RO, Nielsen MS and Olivecrona G: Endocytosis of apolipoprotein A-V by members of the low density lipoprotein receptor and the VPS10p domain receptor families. J Biol Chem 283: 25920-25927, 2008.

14. Farese RV Jr and Walther TC: Lipid droplets finally get a little R-E-S-P-E-C-T. Cell 139: 855-860, 2009.

15. Wolins NE, Brasaemle DL and Bickel PE: A proposed model of fat packaging by exchangeable lipid droplet proteins. FEBS Lett 580: 5484-5491, 2006

16. Brasaemle DL: Thematic review series: Adipocyte biology. The perilipin family of structural lipid droplet proteins: Stabilization of lipid droplets and control of lipolysis. J Lipid Res 48 2547-2559, 2007

17. Souza SC, Muliro KV, Liscum L, Lien P, Yamamoto MT, Schaffer JE, Dallal GE, Wang X, Kraemer FB, Obin M and Greenberg AS: Modulation of hormone-sensitive lipase and protein kinase A-mediated lipolysis by perilipin A in an adenoviral reconstituted system. J Biol Chem 277: 8267-8272, 2002.

18. Tansey JT, Sztalryd C, Gruia-Gray J, Roush DL, Zee JV, Gavrilova O, Reitman ML, Deng CX, Li C, Kimmel AR and Londos C: Perilipin ablation results in a lean mouse with aberrant adipocyte lipolysis, enhanced leptin production, and resistance to diet-induced obesity. Proc Natl Acad Sci USA 98: 6494-6499, 2001.

19. Martinez-Botas J, Anderson JB, Tessier D, Lapillonne A, Chang BH, Quast MJ, Gorenstein D, Chen KH and Chan L: Absence of perilipin results in leanness and reverses obesity in Lepr(db/db) mice. Nat Genet 26: 474-479, 2000.

20. Keller P, Petrie JT, De Rose P, Gerin I, Wright WS, Chiang SH, Nielsen AR, Fischer CP, Pedersen BK and MacDougald OA: Fat-specific protein 27 regulates storage of triacylglycerol. J Biol Chem 283: 14355-14365, 2008.

21. Puri V, Konda S, Ranjit S, Aouadi M, Chawla A, Chouinard M, Chakladar A and Czech MP: Fat-specific protein 27, a novel lipid droplet protein that enhances triglyceride storage. J Biol Chem 282: 34213-34218, 2007.

22. Nishino N, Tamori Y, Tateya S, Kawaguchi T, Shibakusa T, Mizunoya W, Inoue K, Kitazawa R, Kitazawa S, Matsuki Y, et al: FSP27 contributes to efficient energy storage in murine white adipocytes by promoting the formation of unilocular lipid droplets. J Clin Invest 118: 2808-2821, 2008.

23. Toh SY, Gong J, Du G, Li JZ, Yang S, Ye J, Yao H, Zhang Y, Xue B, Li Q, et al: Up-regulation of mitochondrial activity and acquirement of brown adipose tissue-like property in the white adipose tissue of fsp27 deficient mice. PLoS One 3: e2890, 2008

24. Matsusue K: A physiological role for fat specific protein $27 /$ cell death-inducing DFF45-like effector $\mathrm{C}$ in adipose and liver. Biol Pharm Bull 33: 346-350, 2010.

25. Inohara N, Koseki T, Chen S, Wu X and Núñez G: CIDE, a novel family of cell death activators with homology to the $45 \mathrm{kDa}$ subunit of the DNA fragmentation factor. EMBO J 17 : 2526-2533, 1998

26. Magnusson B, Gummesson A, Glad CA, Goedecke JH, Jernås M, Lystig TC, Carlsson B, Fagerberg B, Carlsson LM and Svensson PA: Cell death-inducing DFF45-like effector C is reduced by caloric restriction and regulates adipocyte lipid metabolism. Metabolism 57: 1307-1313, 2008.

27. Beckstead JA, Oda MN, Martin DD, Forte TM, Bielicki JK, Berger T, Luty R, Kay CM and Ryan RO: Structure-function studies of human apolipoprotein A-V: A regulator of plasma lipid homeostasis. Biochemistry 42: 9416-9423, 2003.

28. Gauthier B, Robb M and McPherson R: Cholesteryl ester transfer protein gene expression during differentiation of human preadipocytes to adipocytes in primary culture. Atherosclerosis 142: 301-307, 1999
29. Prawitt J, Niemeier A, Kassem M, Beisiegel U and Heeren J: Characterization of lipid metabolism in insulin-sensitive adipocytes differentiated from immortalized human mesenchymal stem cells. Exp Cell Res 314: 814-824, 2008.

30. Suganami T, Nishida J and Ogawa Y: A paracrine loop between adipocytes and macrophages aggravates inflammatory changes: Role of free fatty acids and tumor necrosis factor alpha. Arterioscler Thromb Vasc Biol 25: 2062-2068, 2005.

31. Suganami T, Tanimoto-Koyama K, Nishida J, Itoh M, Yuan X, Mizuarai S, Kotani H, Yamaoka S, Miyake K, Aoe S, et al: Role of the Toll-like receptor 4/NF-kappaB pathway in saturated fatty acid-induced inflammatory changes in the interaction between adipocytes and macrophages. Arterioscler Thromb Vasc Biol 27: 84-91, 2007.

32. Livak KJ and Schmittgen TD: Analysis of relative gene expression data using real-time quantitative PCR and the 2(-Delta Delta $\mathrm{C}(\mathrm{T})$ ) method. Methods 25: 402-408, 2001

33. Samad F, Pandey M, Bell PA and Loskutoff DJ: Insulin continues to induce plasminogen activator inhibitor 1 gene expression in insulin-resistant mice and adipocytes. Mol Med 6: 680-692, 2000.

34. Rousset S, Alves-Guerra MC, Mozo J, Miroux B, Cassard-Doulcier AM, Bouillaud F and Ricquier D: The biology of mitochondrial uncoupling proteins. Diabetes 53 (Suppl 1): S130-S135, 2004

35. Cederberg A, Grønning LM, Ahrén B, Taskén K, Carlsson P and Enerbäck S: FOXC2 is a winged helix gene that counteracts obesity, hypertriglyceridemia, and diet-induced insulin resistance. Cell 106: 563-573, 2001.

36. Masson O, Chavey C, Dray C, Meulle A, Daviaud D, Quilliot D, Muller C, Valet $\mathrm{P}$ and Liaudet-Coopman E: LRP1 receptor controls adipogenesis and is up-regulated in human and mouse obese adipose tissue. PLoS One 4: e7422, 2009.

37. de Ferranti S and Mozaffarian D: The perfect storm: Obesity, adipocyte dysfunction, and metabolic consequences. Clin Chem 54: 945-955, 2008

38. Ko KW, Avramoglu RK, McLeod RS, Vukmirica J and Yao Z: The insulin-stimulated cell surface presentation of low density lipoprotein receptor-related protein in 3T3-L1 adipocytes is sensitive to phosphatidylinositide 3-kinase inhibition. Biochemistry 40: 752-759, 2001.

39. Corvera S, Graver DF and Smith RM: Insulin increases the cell surface concentration of alpha 2-macroglobulin receptors in 3T3-L1 adipocytes. Altered transit of the receptor among intracellular endocytic compartments. J Biol Chem 264: 10133-10138, 1989.

40. Sawada T, Miyoshi H, Shimada K, Suzuki A, Okamatsu-Ogura Y, Perfield JW II, Kondo T, Nagai S, Shimizu C, Yoshioka N, et al: Perilipin overexpression in white adipose tissue induces a brown fat-like phenotype. PLoS One 5: e14006, 2010.

41. Busiello RA, Savarese S and Lombardi A: Mitochondrial uncoupling proteins and energy metabolism. Front Physiol 6: 36, 2015.

42. Nicholls DG and Locke RM: Thermogenic mechanisms in brown fat. Physiol Rev 64: 1-64, 1984

43. Kopecky J, Clarke G, Enerbäck S, Spiegelman B and Kozak LP: Expression of the mitochondrial uncoupling protein gene from the aP2 gene promoter prevents genetic obesity. J Clin Invest 96 2914-2923, 1995.

44. Tiraby C, Tavernier G, Lefort C, Larrouy D, Bouillaud F, Ricquier D and Langin D: Acquirement of brown fat cell features by human white adipocytes. J Biol Chem 278: 33370-33376, 2003.

45. Si Y, Palani S, Jayaraman A and Lee K: Effects of forced uncoupling protein 1 expression in 3T3-L1 cells on mitochondrial function and lipid metabolism. J Lipid Res 48: 826-836, 2007.

46. Oberkofler H, Dallinger G, Liu YM, Hell E, Krempler F and Patsch W: Uncoupling protein gene: Quantification of expression levels in adipose tissues of obese and non-obese humans. J Lipid Res 38: 2125-2133, 1997.

47. Yang X, Enerbäck S and Smith U: Reduced expression of FOXC2 and brown adipogenic genes in human subjects with insulin resistance. Obes Res 11: 1182-1191, 2003. 